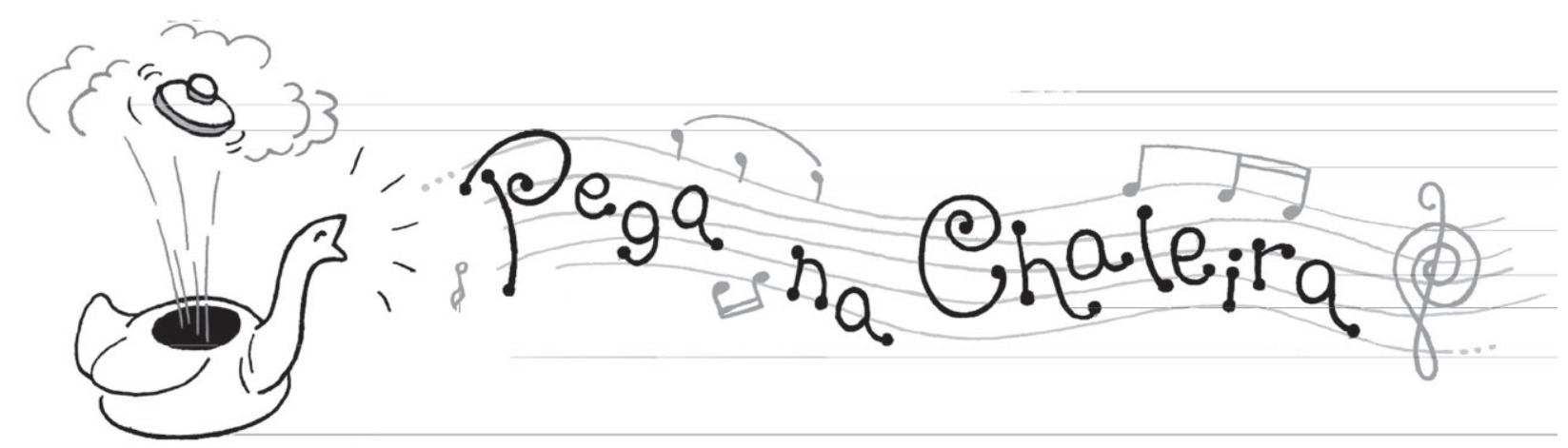

PEGA NA CHALEIRA - RESENHAS

\title{
Una propuesta de discusión acerca de las cuestiones teórico-metodológicas en el estudio del rock nacional argentino
}

\author{
A proposal for the discussion of theoretical and methodological questions in the study of rock \\ nacional argentino
}

Mara Favoretto (University of Melbourne, Melbourne, Austrália)

mara.f@unimelb.edu.au

GARCÍA, Miguel A. (org.). Rock en papel: bibliografía crítica de la producción académica sobre el rock en Argentina. La Plata, Editorial de la Universidad Nacional de La Plata, 2010, 312 p.

Rock en papel es una iniciativa interesante y ciertamente útil para todos aquellos interesados en el rock como objeto de estudio académico. Su propósito, según lo aclara su compilador Miguel A. García, es "comprender cómo el rock local fue definido en tanto objeto de estudio y cómo fue evaluado en términos sociales y estéticos, con las lentes de las ciencias sociales y las humanidades, y a partir de los gustos personales de sus exégetas" (GARCIA, 2010, p.13). Se trata de una serie de ensayos, a cargo de un equipo de investigadores dirigidos por Garcia, que promueven una discusión sobre las temáticas que se desarrollan en los 52 trabajos que estos ocho autores reseñan en Rock en papel. Es de lamentar que la única biografía que se ofrece es la del director del proyecto, ya que no figuran datos más allá del nombre de los reseñadores y autores de los ensayos con que se inicia el libro.
El primero de estos ensayos, firmado por Miguel A. García, explora las estrategias narrativas que se suelen emplear para el análisis de la música popular. García considera que "el involucramiento afectivo del observador con su objeto de estudio" suele ser un problema y que algunos críticos y musicólogos "en general tienden a utilizar un lenguaje técnico y argumentativo para sostener la superioridad de la música que defienden y la inferioridad de la música que critican" (GARCIA, 2010, p. 24). García afirma que la escritura descentrada y desnaturalizante, en los textos reseñados, es la más difícil de encontrar. Acertadamente destaca la necesidad de un discurso académico que reconozca la variedad de paradigmas estéticos existentes sin compararlos desde la 'superioridad de ninguno de ellos' (GARCIA, 2010, p. 27). 
El segundo ensayo, a cargo de Claudio G. Castro, de índole descriptiva, realiza un recorrido por algunos de los trabajos incluidos en esta bibliografía crítica estableciendo qué lugar ocupan las letras de las canciones en la metodología empleada en cada caso. Advierte que un enfoque textualizado, en el que la fuente primaria de análisis está constituida precisamente por los textos, "acaba por monopolizar la interpretación del sentido de las letras al no dar cabida a las voces de los actores" (GARCIA, 2010, p. 30).

Camila Juárez plantea que varios de los autores incluidos en la bibliografía seleccionada excluyen consideraciones de tipo musical y enfocan su análisis en cuestiones sociales, identitarias, y factores políticos y económicos. Sin embargo, reconoce que debido a la naturaleza ecléctica del género, se hace dificultosa su definición musical (GARCIA, 2010, p. 40). Juárez convoca a la musicología a incursionar en el análisis de rock como género.

Lisa Di Cione coincide con Juárez en que prevalecen aquellos trabajos

"que centrados prioritariamente en aspectos literarios, históricos, sociales, culturales o estéticos, evitan el trato directo con el material sonoro" (GARCIA, 2010, p. 47). Di Cione reconoce la dificultad que presenta el rock como género musical a la hora de ensayar un modelo de análisis adecuado y, al igual que sus colegas, sostiene la necesidad de nuevos análisis académicos que tengan en cuenta los aspectos de orden musical.

Laura Novoa se suma a esta premisa, destacando la importancia y necesidad de incluir en el análisis formal aquellos elementos sonoros y musicales que se ponen en juego en el estudio de grabación. Considera fundamental tal práctica "para comprender ciertas dinámicas que son determinantes dentro del campo de la música popular actual" (GARCIA, 2010, p. 56). Destacando un congreso organizado en Londres en el 2005 a tales fines ${ }^{1}$, Novoa insiste en que la musicología local debería prestar atención similar al tratamiento del sonido grabado.

Probablemente el ensayo que realiza la lectura más atinada de la bibliografía bajo la lupa en este trabajo es el de Francisco Levaggi. En él reconoce que todos los autores en cuestión coinciden en afirmar que el rock en Argentina surgió a partir de una tensión, de un conflicto y un rechazo, aunque advierte que no se ha prestado la suficiente atención al modo en que dicha tensión se traslada al público (GARCIA, 2010, p. 64). Señala también, acertadamente, que todos los autores destacan la Guerra de Malvinas como instancia decisiva en la incorporación definitiva del rock al mercado discográfico argentino.

Juliana Guerrero intenta indagar cómo se narra la historia del rock en los trabajos reseñados, repitiendo al igual que en los ensayos anteriores, que queda pendiente un abordaje riguroso de los aspectos exclusivamente musicales en la observación de los cambios experimentados por el género históricamente (GARCIA, 2010, p. 66). Luego de un análisis detallado, concuerda con Levaggi en que en todos los casos, el hito histórico en el que todos estos trabajos convergen es la Guerra de Malvinas.

A Karen Avenburg le preocupa que el uso de entrevistas aparece limitado en los trabajos abordados, ya que las considera una fuente relevante cuya inclusión debe ser debidamente articulada a fin de evitar la anulación de voces. Sin embargo, en la reseña de uno de los trabajos del sociólogo Pablo Vila -uno de los primeros en analizar el movimiento rock nacional académicamenteNovoa destaca que "la base empírica del artículo está conformada por [...] entrevistas [...] y cartas de lectores" (GARCIA, 2010, p. 298).

Luego de los ocho ensayos mencionados, el libro incluye 52 recensiones de trabajos, de las cuales 14 corresponden a ponencias en congresos, 16 a artículos en revistas especializadas, 5 a libros, 14 a capítulos en compilaciones y 3 a tesis inéditas. Resulta sorprendente que una de estas tres tesis inéditas reciba una reseña de 18 páginas -notoriamente mucho más extensa que cualquiera de las otras 51-y que, además, no sea posible acceder a una copia de dicha tesis sin gran dificultad (GARCIA, 2010, p.108). ${ }^{2} \mathrm{El}$ lector interesado encontrará esta reseña de poca utilidad si no le es posible tener acceso al trabajo original, quizás localizable físicamente sólo en la Universidad de Buenos Aires, donde fue presentada originalmente.

Si bien García aclara que el proceso de selección de trabajos a reseñar presentó gran complejidad debido a la existencia de una considerable variedad de aproximaciones y metodologías, es notable la ausencia de algunos autores clave en el análisis del rock nacional argentino, como es el caso de Oscar CONDE (2007) y sus Poéticas del rock o el trabajo de Miguel GRINBERG (1977), que, aunque de indole periodística, ha sido pieza fundamental en la historiografía del rock argentino y base de investigaciones posteriores.

Como se ha señalado, en reiteradas ocasiones en Rock en papel, sus autores destacan que en la gran mayoría de los trabajos analizados se hace mayor hincapié en elementos no musicales, como los aspectos de orden social, lingüístico, político y económico. A la vez plantean el cuestionamiento acerca de cuáles deberían ser las herramientas especificas para el análisis del rock. Rock en papel constituye una reflexión sobre las perspectivas teórico-metodológicas que se han empleado al estudiar el rock nacional argentino y un Ilamado a ampliar estas perspectivas, incluyendo aspectos que han sido dejados de lado. En concreto, y esto es quizás el mayor aporte del libro, sus autores enfatizan la necesidad de un abordaje musicológico que se articule con las disciplinas ya utilizadas. Sin duda, un reclamo válido y una iniciativa que debería ser aplaudida y atendida sin mayores demoras. 


\section{Referencias}

CONDE, Oscar (ed.) Poéticas del rock. Volumen 1. Buenos Aires: Marcelo Héctor Olivetti Editor, 2007.

GARCíA, Miguel A. (ed.). Rock en papel. Bibliografía crítica de la producción académica sobre el rock en Argentina. La Plata, Editorial de la Universidad Nacional de La Plata, 2010, 312 p.

GRINBERG, Miguel. La música progresiva argentina (cómo vino la mano). Buenos Aires: Editorial Convergencia, 1977. Reeditado en 1985 (Editorial Mutantia), 1993 (Editorial Distal) y 2008 (Gourmet Musical Ediciones).

\section{Notas}

1 Los organizadores de dicho congreso fueron el CHARM (Centre for the History and Analysis of Recorded Music) y el London College of Music \& Media, Thames Valley University (GARCIA, 2010, p. 57).

2 Se trata de la tesis de CITRO, Silvia. Cuerpos festivo rituales: un abordaje desde el rock. Tesis de Licenciatura en Ciencias Antropológicas, Facultad de Filosofía y Letras, Universidad de Buenos Aires. Inédita. 233 páginas, 1997.

Mara Favoretto is a Lecturer in Spanish and Latin American Studies at the University of Melbourne, Australia, where she coordinates and teaches a range of Language and Culture Studies subjects. She specializes in contemporary popular culture and popular music lyrics as counter discourse in cultural crisis. Her research interests include rhetorical tropes used to encode subversive messages under the constraints of censorship and cultural expressions of resistance in Latin American society. She is the author of Alegoría e ironía bajo censura en la Argentina del Proceso (New York: Edwin Mellen Press, 2010) 